\title{
FIBROUS CAVITY IN AN IMMUNOCOMPROMISED LUNG: NOCARDIOSIS MASQUERADING AS TUBERCULOSIS
}

\section{KARTHIK RAO N ${ }^{1}$, NAVIN PATIL ${ }^{2 *}$, NEHA AGARWAL ${ }^{1}$, HANDATTUMANJUNATHA HANDE ${ }^{1}$, AMY JOSEPH ${ }^{1}$, GEORGE VARGHESE ${ }^{3}$, RAGHAVENDRA RAO ${ }^{1}$}

${ }^{1}$ Department of Medicine, Kasturba Medical College, Manipal University, India. ${ }^{2}$ Department of Pharmacology, Kasturba Medical College, Manipal University, India. ${ }^{3}$ Kasturba Medical College, Manipal University, India. Email: navin903@gmail.com

Received: 02 July 2016, Revised and Accepted: 11 July 2016

\section{ABSTRACT}

Nocardia species can cause fulminant necrotizing cavitary pneumonia. Diagnosis involves identification of the organism, speciation, and correlating with appropriate clinical and radiological findings. Speciation is often difficult due to the complexities involved in culturing the organism. Treatment involves administering antibiotics for a prolonged duration. The authors hereby report a case of pulmonary nocardiosis in a patient who had premorbidities such as cirrhosis of the liver and membrano-proliferative glomerular nephritis on immunosuppression with high dose corticosteroids making him vulnerable to the organism.

Keywords: Nocardia, Pneumonia, Actinomycetes, Lung.

(C) 2016 The Authors. Published by Innovare Academic Sciences Pvt Ltd. This is an open access article under the CC BY license (http://creativecommons. org/licenses/by/4. 0/) DOI: http://dx.doi.org/10.22159/ajpcr.2016.v9i6.13855

\section{BACKGROUND}

Nocardia is a group of Gram-positive aerobic actinomycetes that are most commonly found in the soil. They most often affect immunocompromised people, e.g., those on long-term glucocorticoid therapy, AIDS, post-transplantation, and lymphoma [1]. The disease is transmitted by inhalation of fragmented bacteria mycelia resulting in either pulmonary or disseminated disease. $50 \%$ of pulmonary disease is found in the form of pneumonia.

The clinical picture of nocardiosis resembles pulmonary tuberculosis (TB). Hence, the diagnosis may be delayed if the clinician and the microbiologist do not suspect the disease [2]. We hereby present a case of pulmonary nocardiosis that mimicked pulmonary TB in a patient with pre-morbidities of liver cirrhosis and membrano-proliferative glomerulonephritis on long-term glucocorticoid therapy.

\section{CASE REPORT}

A 38-year-old male patient recently diagnosed to have cirrhosis of the liver (cryptogenic) and primary membrano-proliferative glomerulonephritis on high dose corticosteroids $(1 \mathrm{mg} / \mathrm{kg}$ ) presented with symptoms of a cough, fever, and pleuritic type of chest pain of 3 weeks duration. Clinical examination revealed coarse crackles in the right lung base. Salient routine investigations showed hemoglobin of $10.4 \mathrm{~g} / \mathrm{dL}$, leukocytosis of $20,900 / \mu \mathrm{L}$, and extracytoplasmic stress response of $57 \mathrm{~mm} / \mathrm{hrs}$. A right hilar homogenous opacity was seen on chest radiography (Fig. 1).

Empirical treatment for severe community-acquired pneumonia with intravenous piperacillin and tazobactam was administered. As the patient had history prolonged hospital stay before this presentation, possibility of hospital-acquired pneumonia was considered but was ruled out, as cultures did not show growth of any pathological bacteria.

Due to worsening clinical picture, a contrast-enhanced computed tomography (CECT) scan of thorax was obtained, which showed multiple nodules (5-10 mm) diffusely scattered in both lobes, few of them showing central cavitation, and large consolidatory patches with areas of breakdown and cavitation in anterior segment of right upper lobe and superior lingular segment of left upper lobe (Fig. 2). Pulmonary TB

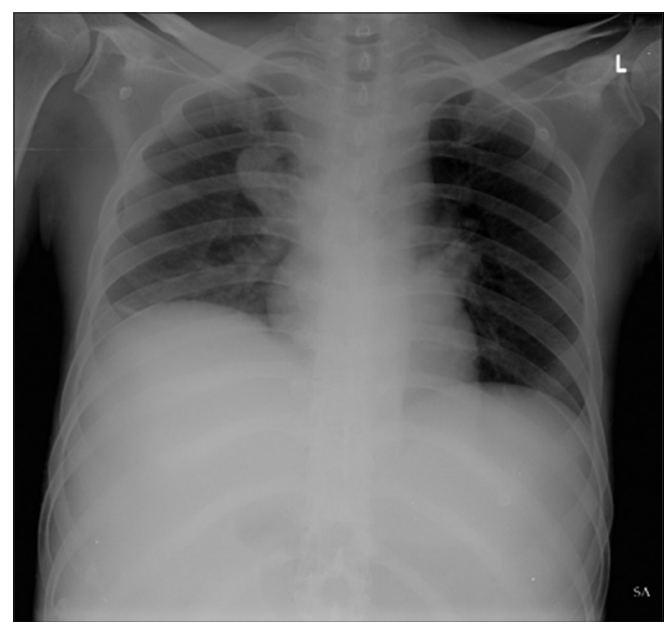

Fig. 1: Chest X-ray showing opacity in the right lung

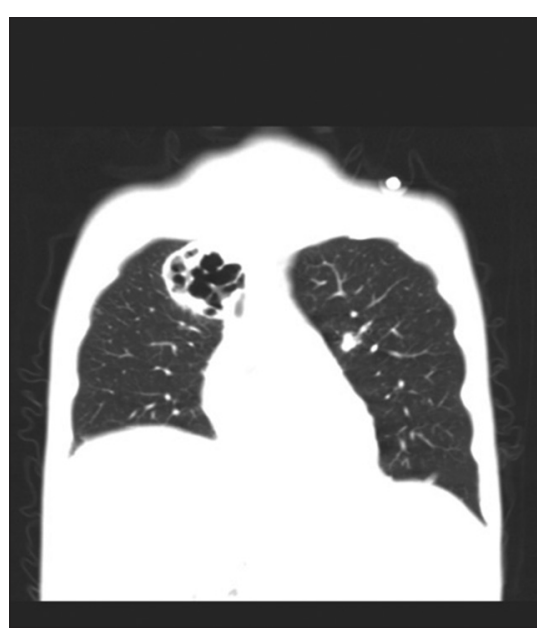

Fig. 2: Contrast-enhanced computed tomography thorax showing multiple nodules and cavitation 


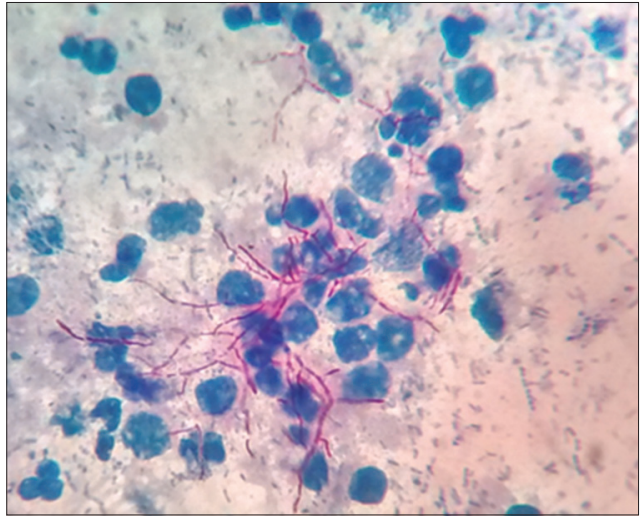

Fig. 3: Histopathology showing acid-fast bacilli

was suspected as the patient was on immunosuppression therapy and presented with history of low-grade fever and prolonged cough. It was ruled out as repeated sputum samples and fluid from bronchoalveolar lavage sent for staining of acid-fast bacilli tested negative despite chest radiography revealing features of the cavity. After discussing the clinical and radiological scenario with the microbiologist, a possibility of nocardiosis was considered. A modified acid-fast staining was performed that revealed acid-fast filamentous branching rods suggestive of Nocardia (Fig. 3). However, further speciation of Nocardia was not possible due to lack of relevant facilities.

Ideally, the treatment of nocardiosis is to be done following speciation of the organism and decided accordingly. The most common species are Nocardia asteroides sensu stricto, Nocardia farcinica, Nocardia nova, and Nocardia transvalensis complex. According to the species, the organism has varying susceptibility to trimethoprim-sulfamethoxazole, amikacin, third generation cephalosporins, linezolid, and imipenem. The first line therapy that is usually used is the trimethoprimsulfamethoxazole combination. However, in severe infections, empirical therapy with 2-3 drugs should be started [2]. Treatment should ideally be for 1 year in immunocompromised individuals. As speciation was not possible in this facility, the subject of this discussion was empirically started on meropenem, amikacin, and sulfamethoxazole-trimethoprim. Monitoring of the effect of the treatment was done both clinically and radiologically. After the initiation of treatment, there was a remarkable improvement in the patient's symptoms. After a month of therapy, there was a significant improvement in clinical and radiological. The patient will further be followed up monthly for 1 year.

\section{DISCUSSION}

Nocardia is a Gram-positive aerobic actinomycete which results in diseases ranging from pulmonary to central nervous system (CNS) to topical, etc., [3]. It is most commonly seen in immunocompromised individuals; in adults more than pediatric age group and in males more than females. The subject of this discussion is a 38-year-old male, with pre-morbidities of cirrhosis of the liver and membrano-proliferative glomerular nephritis, on long-term glucocorticoid therapy who presented with the pleuritic type of chest pain and cough.
Diagnosis of Nocardia is done by isolating and identifying the organism from a clinical specimen. Nocardia may be isolated from the sputum, blood, or granules in cases of cutaneous infection, but it is usually very difficult [4]. In suspected pulmonary cases, bronchoalveolar lavage or lung biopsy may be useful. In this patient, initially, X-ray showed a hilar shadow. CECT scan of the thorax showed multiple nodules (5-10 mm) diffusely scattered in both lobes, few of them showing central cavitation, and large consolidatory patches with areas of breakdown and cavitation in the anterior segment of the right upper lobe and superior lingular segment of left upper lobe. Suspecting $\mathrm{TB}$, acid-fast staining of the sputum was done. Due to the branching structure seen, modified acid-fast staining was done which revealed Nocardia spp. To make diagnosis of Nocardia via staining methods, modified acid-fast staining should be done [5]. CT chest findings of pulmonary nocardiosis can range from pulmonary nodules to cavitary pneumonia [4].

Ideally, so as to tailor the treatment for the specific organism, speciation of the organism isolated from the patient should be performed. However, as the facility was not available in our setup, the patient was empirically started on amikacin, trimethoprim-sulfamethoxazole, and meropenem. The duration of therapy has not been standardized; however, in immunocompromised individuals, prolonged therapy is advised. In isolated cutaneous infection, the patient is treated for a minimum of 3-6 months and a maximum of 6-12 months. For serious infections, i.e., serious pulmonary infections, CNS infections, or disseminated disease, immunocompromised patients should be treated for at least a year. Following the start of treatment, the patient had improved clinically.

\section{CONCLUSION}

Diagnosis of nocardiosis is a challenge. The case treating clinician and the microbiologist must have a high index of suspicion for the prompt diagnosis of this condition. In case of immunocompromised individuals, nocardial infections should always be kept in mind, especially when radiological findings mimic $\mathrm{TB}$, but repeated sputum examinations are negative for TB bacilli. The case highlights the importance of early diagnosis, appropriate antibiotics for the appropriate duration that led to the successful management in this gentleman.

\section{REFERENCES}

1. Lederman ER, Crum NF. A case series and focused review of nocardiosis: Clinical and microbiologic aspects. Medicine (Baltimore) 2004;83(5):300-13.

2. Ameen M, Arenas R, Vásquez del Mercado E, Fernández R, Torres E, Zacarias R. Efficacy of imipenem therapy for Nocardia actinomycetomas refractory to sulfonamides. J Am Acad Dermatol 2010;62(2):239-46.

3. Jayaprakash B, Rao K, Patil N, Avinash A, Rao R, Kunder SK, et al. Nocardia meningitis in a newly diagnosed HIV positive patient. Res J Pharm Biol Chem Sci 2016;7(1):1575-7.

4. Brown-Elliott BA, Brown JM, Conville PS, Wallace RJ Jr. Clinical and laboratory features of the Nocardia spp. Based on current molecular taxonomy. Clin Microbiol Rev 2006;19(2):259-82.

5. Kumar A, Reddy A, Satagopan K. Unusual presentation of pulmonary nocardiosis as pyopneumothorax in HIV. Lung India 2015;32(3):295-6. 